\title{
Persistent Antigens Hypothesis: The Human Leukocyte Antigen (HLA) Connection
}

\author{
Lisa M. James ${ }^{1,2,3}$, Apostolos P. Georgopoulos $1,2,3,4 *$ \\ 'Brain Sciences Center, Department of Veterans Affairs Health Care System, Minneapolis, MN, 55417, USA \\ ${ }^{2}$ Department of Neuroscience, University of Minnesota Medical School, Minneapolis, MN 55455, USA \\ ${ }^{3}$ Department of Psychiatry, University of Minnesota Medical School, Minneapolis, MN 55455, USA \\ ${ }^{4}$ Department of Neurology, University of Minnesota Medical School, Minneapolis, MN 55455, USA
}

Article Info

\section{Article Notes}

Received: December 1, 2018

Accepted: December 24, 2018

\section{${ }^{*}$ Correspondence:}

Dr. Georgopoulos AP, Brain Sciences Center (11B), Minneapolis VAHCS, One Veterans Drive, Minneapolis, MN 55417, USA

E-mail: omega@umn.edu.

C) 2018 Georgopoulos AP. This article is distributed under the terms of the Creative Commons Attribution 4.0 International License

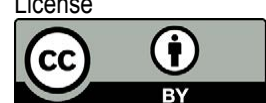

\section{Human Leukocyte Antigen (HLA) Overview}

HLA genes play a critical role in immune protection from foreign antigens including viruses, bacteria, and parasites ${ }^{1}$. Located in the Major Histocompatibility Complex (MHC) of chromosome 6, HLA genes code for glycoproteins that exist on the surface of most cells in order to facilitate immune surveillance and initiate an immune response to eliminate foreign antigens. There are two main classes of HLA (Class I and Class II) that support the elimination of cytosolic or extracellular foreign antigens through cell destruction and antibody production, respectively. HLA genes have evolved to be the most highly polymorphic in the human genome, thereby maximizing species resistance to foreign antigens and promoting survival. Nonetheless, successful elimination of foreign antigens is predicated on a match between one's HLA and epitopes derived from foreign antigen proteins. Each person has a limited repertoire of HLA proteins inherited in a Mendelian fashion for each class. Fortunately, each HLA protein can match with various epitopes and, since everyone has one or two alleles at each of the classical loci (Class I HLA-A, B, and C and Class II HLA-DP, DQ, and DR), a large number of antigens can be effectively eliminated.

\section{The Match}

The structure of the HLA molecule determines the specific epitopes thatcanbind and, therefore, beeliminated. Although theoverallstructure of Class I and Class II HLA molecules differ, they are similar in that for both classes polypeptide chains form a binding groove. It is variation within the binding groove that contributes to high polymorphism. For instance, within the Class II HLA DRB $1 * 13$ allele family alone there are 330 known variants ${ }^{2}$, although some alleles such as DRB1*13:01 and DRB1*13:02 are much more common than others forms of the allele. DRB1*13:01 and DRB1*13:02 differ by a single amino acid in the $\beta$-chain; yet that difference has been shown to significantly alter the electrostatic properties of the binding groove ${ }^{3}$. Indeed, seemingly small differences conferred by single amino acid substitutions result in variations in charge, stability, and binding affinity ${ }^{4}$ that translate into the differential ability to effectively eliminate foreign antigens. Those differences are amplified across different genes and even more so across HLA classes. Class I HLA molecules, which are expressed on nucleated cells, present small peptides (8-10 amino acid residues) from proteolytically degraded intracellular viruses, bacteria, and tumors to cytotoxic T cells for degradation. The process is facilitated by transport of the cytosolic peptides to the endoplasmic reticulum where they 
are loaded onto Class I molecules and exported to the cell surface for presentation to CD8+ cytotoxic T cells for cell destruction. Class II HLA, which are expressed on lymphocytes and antigen presenting cells including macrophages, dendritic cells, and monocytes, present larger peptides (12-20 amino acid residues) derived from endocytosed exogenous antigens including viruses and bacteria to $\mathrm{CD} 4+\mathrm{T}$ cells which stimulate the production of antibodies. For Class II molecules, antigen binding is facilitated by Cathepsin $S$ which removes an invariant chain that blocks binding. Despite all of these differences, Class I and Class II HLA share the same overarching goal and work in concert to maximize elimination of foreign antigens.

\section{HLA-Disease Associations}

The HLA region of the genome is associated with the greatest number of human diseases ${ }^{5}$. Given its role in immune system functioning it is not surprising that HLA has been implicated in a host of autoimmune and infectious diseases ${ }^{6}$; however, more recent studies have also implicated HLA in diseases ranging from schizophrenia $^{7}$ and autism ${ }^{8}$ to neurodegenerative diseases such as Alzheimer's disease ${ }^{9,10}$ and Parkinson's disease ${ }^{11}$. Reports of HLA-disease associations have typically been derived from population studies in which certain genes are found to be more prevalent in specific disease groups. This particular approach of identifying risk-related genes is complicated by high linkage disequilibrium. Due to high linkage disequilibrium within the HLA region, some combinations of genes, referred to as haplotypes, are inherited together thereby leading to potential "mistaken identity" in terms of disease risk ${ }^{12}$.

Etiopathological models underlying HLA-disease associations have been summarized elsewhere ${ }^{5,6}$. Briefly, prevailing models implicate non-mutually exclusive processes including alterations in $\mathrm{T}$ cell repertoire, molecular mimicry, aberrant antigen recognition, and ineffective interactions of the antigen-HLA complex with $\mathrm{T}$ cells as promoting disease susceptibility. A multitude of viral escape mechanisms may also contribute to diseases via disruption of immune system functioning ${ }^{13,14}$ Despite extensive study of mechanisms underlying HLAdisease associations, the fact remains that in most cases the mechanisms remain poorly understood and likely represent genetic vulnerability coupled with environmental insults $^{6,15}$. Further, as compellingly discussed elsewhere ${ }^{12}$, most prevailing theories of HLA-disease association are inconsistent with the biology, epidemiology, and evolution of HLA molecules.

\section{HLA Protective Effects}

Given the biological and evolutionarily adaptive role of HLA in the clearance of foreign antigens and maintenance of immune system functioning, the concept of HLA-disease associations is counterintuitive. That is, HLA's primary function is host protection. Accordingly, there also exists robust evidence of HLA protective effects. To that end, protective HLA alleles have been observed in conditions including autoimmune disorders ${ }^{6,16}$, HIV $^{17,18}$, Hepatitis B and $\mathrm{C}^{19}$, malaria ${ }^{20}$, and Gulf War Illness ${ }^{15,21}$, among others. Furthermore, certain HLA alleles have been shown to exert protective effects in healthy individuals, minimizing age-related brain changes typically attributed to "normal" $\operatorname{aging}^{22,23}$ (see below).

In many cases, protective effects have been shown to vary by population ${ }^{19}$. HLA is known to vary by ethnicity and/or locale, presumably reflecting evolutionary adaptations related to population differences in pathogen exposure. Nonetheless, some alleles appear to more broadly promote protection across a wide variety of diseases and populations. For example, HLA-DRB1*13 has been shown to be protective against Hepatitis $B$ and $\mathrm{C}^{19}$ and various autoimmune conditions ${ }^{16,24}$ across several populations. Similarly, variants of the DRB $1 * 13$ allele group have been shown to protect against the development of Gulf War Illness ${ }^{15}$, and protect against brain atrophy in Gulf War veterans ${ }^{20}$. Furthermore, the protective effects of DRB1*13 have been observed in cognitively healthy women with carriers evidencing minimal age-related brain atrophy and functional brain changes relative to non-carriers ${ }^{22,23}$. Of note, within the DRB $1 * 13$ allele family, variations at the protein level confer differential protection. For example, while DRB1*13:02 has been associated with broad protective effects, DRB1*13:01, which differs by only a single amino acid residue, has shown both protective $\mathrm{e}^{23,25}$ and risk effects ${ }^{3,26}$. The differential effects are presumably associated with changes in the binding groove that affect antigen binding and subsequent elimination.

\section{An alternative: Persistent Antigen Hypothesis}

In light of the biological role of HLA in immune system surveillance and regulation coupled with observed HLA-associated disease protection, we have proposed an alternative model of disease susceptibility referred to as the Persistent Antigen hypothesis. Essentially, maintenance of immune system functioning rests on successful elimination of foreign antigens, a process that partially depends on a match between the antigen and an individual's HLA composition. In contrast, the absence of an antigen-HLA match prevents the elimination of the antigen. Consequently, a persistent antigen may lead to inflammation, cell damage, autoimmunity ${ }^{27}$, and atrophy ${ }^{21,22}$. The Persistent Antigen hypothesis contends that the HLAantigen match is a critical step for the elimination of foreign antigens and health maintenance; in the absence of an HLA-antigen match, any number of downstream processes involved in foreign antigen recognition, cell 
destruction, and antibody production would be disrupted including those specifically implicated in HLA-disease associations $^{5,6}$. Thus, the Persistent Antigen hypothesis, which highlights the fundamental role of the HLA-antigen match, is complementary to other etiopathological models of HLA-disease associations.

Our research on Gulf War Illness (GWI) provides an illustrative example. GWI is a neuroimmune condition ${ }^{28}$ of uncertain etiology that has affected one-third of US veterans deployed to the 1990-1991 Gulf War ${ }^{29}$. GWI is characterized by diffuse, chronic symptoms including fatigue, joint and muscle pain, and neurocognitive and mood disruptions, as well as gastrointestinal, respiratory, and skin problems ${ }^{30,31}$. Given the overlap of GWI symptoms with known autoimmune conditions and evidence of abnormal immune responses in veterans with $\mathrm{GWI}^{32-34}$, we investigated HLA involvement with GWI. We identified 6 HLA alleles that were present in healthy GW veterans but were absent or significantly less frequent in those with GWI - that is, GWI was associated with genetic lack of HLA protection ${ }^{15}$. We hypothesized that this genetic vulnerability coupled with environmental hits including multiple, often concurrent vaccinations and/or chemical or other toxic exposures that have been implicated in $\mathrm{GWI}^{27}$ underlie the development of GWI and explain why one-third of veterans were affected whereas the remainder were not. That is, the former may not have been able to effectively eliminate pathogens due to an insufficient match between the pathogenic epitopes and HLA proteins whereas the latter was afforded genetic protection that presumably facilitated effective elimination of pathogens derived from the various environmental exposures that were associated with service during the Gulf War ${ }^{27}$.

Notably, HLA proteins are expressed on most cells (Class I on nucleated cells and Class II on professional antigen presenting cells), providing widespread protection in the case of HLA-antigen matching; however, the absence of HLA-antigen matching in GWI may inflict widespread damage. Indeed, multiple systems and organs are involved in GWI including the brain, lungs, gastrointestinal tract, and musculoskeletal systems. Regarding GWI effects on the brain, infection, autoimmunity, and stress have been shown to result in loss of the blood-brain barrier integrity ${ }^{35}$, which may permit entry of circulating pathogens into the brain, ultimately leading to several GWI brain-related symptoms including fatigue, pain, and neurocognitive mood symptoms. We have also shown that GWI is associated with significant brain atrophy, particularly involving subcortical structures ${ }^{36}$. Remarkably, the presence of protective HLA has been shown to protect against brain atrophy in Gulf War veterans ${ }^{21}$.

The implications of the Persistent Antigen hypothesis extend well beyond Gulf War Illness. In fact, we have recently demonstrated HLA protective effects on maintaining healthy cognitive function across the lifespan.
Age-related changes in brain structure and function have been widely investigated and reviewed elsewhere ${ }^{37}$. For example, age-related brain atrophy at the rate of $2-5 \%$ volume loss per decade beginning around age 40 has been firmly established ${ }^{38}$. However, we recently showed HLA protection against age-related brain atrophy in cognitively healthy women ${ }^{22}$. Remarkably, we also demonstrated HLA protection against changes in brain functioning in cognitively healthy women, even in the presence of apolipoprotein E4, a well-known Alzheimer's disease risk gene ${ }^{23}$. Thus, we hypothesize that efficient HLAantigen matching protects the brain (and other organs) from what has typically been referred to as "normal" agerelated deterioration. In contrast, the absence of an HLAantigen match may hinder elimination of foreign antigens that might otherwise result in subtle, accumulative damage such as that ascribed to cellular senescence ${ }^{39,40}$ and ultimately neurodegenerative diseases including Alzheimer's disease.

\section{Conclusion}

The Persistent Antigen hypothesis provides a novel perspective on HLA-disease associations that is firmly rooted in the biological role of HLA in eliminating foreign antigens via HLA-antigen matching. Thus, the Persistent Antigen hypothesis suggests a plausible mechanism underlying a multitude of diseases in which immune and inflammatory processes are implicated - that is, lack of HLA-antigen matching contributes to antigen persistence which ultimately leads to disease. This, of course, suggests that both exposures to foreign antigens and genetic vulnerability that precludes their elimination are integral in disease development. On the other hand, limited exposure to foreign antigens and/or genetic protection may limit disease and enhance longevity. Indeed, we suspect that these very factors contribute to the enhanced longevity observed in several isolated communities referred to as the "blue zones"41. Finally, the Persistent Antigen hypothesis also implies potential treatment avenues along the lines of personalized precision immunotherapy aimed at facilitating the elimination of circulating persistent antigens which we are currently pursuing ${ }^{42,43}$.

\section{Acknowledgments}

The contents do not represent the views of the U.S. Department of Veterans Affairs or the United States Government. This work was supported by a grant from the United States Department of Defense (award number W81XWH-15-1-0520).

\section{References}

1. Meuer SC, Hussey RE, Hodgdon JC, et al. Surface structures involved in target recognition by human cytotoxic T lymphocytes. Science. 1982; 218: 471-473.

2. Robinson J, Halliwell JA, Hayhurst JH, et al. The IPD and IPD-IMGT/ 
HLA Database: allele variant databases. Nucleic Acids Research 2015; 43: D423-431, version 3.34.0, release date 10-18-18. Retrieved November 15, 2018.

3. Hov JR, Kosmoliaptsis V, Traherne JA, et al. Electrostatic modifications of the HLA-DR P9 peptide-binding pocket and susceptibility to primary sclerosing cholangitis. Hepatology. 2011; 53: 1967-1976.

4. Stern LJ, Brown JH, Jardetzky TS, et al., Crystal structure of the human class II MHC protein HLA-DR1 complexed with an influenza virus peptide. Nature. 1994; 368: 215-221.

5. Trowsdale J, Knight JC. Major histocompatibility complex genomics and human disease. Ann Rev Genom Hum Genet. 2013; 14: 301-323.

6. Gough SC, Simmonds MJ. The HLA region and autoimmune disease: Associations and mechanisms of action. Curr Genomics. 2007; 8(7): 453-65.

7. Brucato N, Guadalupe T, Franke B, et al. A schizophrenia-associated HLA locus affects thalamus volume and asymmetry. Brain Behav Immun. 2015; 46: 311-8.

8. Torres AR, Maciulis A, Stubbs EG, et al. The transmission disequilibrium test suggests that HLA-DR4 and DR13 are linked to autism spectrum disorder. Hum Immunol. 2002; 63(4): 311-6.

9. Lambert JC, Ibrahim-Verbaas CA, Harold D, et al. Meta-analysis of 74,046 individuals identifies 11 new susceptibility loci for Alzheimer's disease. Nat Genet. 2013; 45: 1452-1458.

10. Steele NZ, Carr JS, Bonham LW, et al. Fine-mapping of the human leukocyte antigen locus as a risk factor for Alzheimer disease: a casecontrol study. PLOS 2017, 10.1371/journal.pmed.1002272.

11. Nalls MA, Plagnol V, Hernandez DG, et al. International Parkinson Disease Genomics Consortium Imputation of sequence variants for identification of genetic risks for Parkinson's disease: a meta-analysis of genome-wide association studies. Lancet. 2011; 377: 641-649.

12. Holoshitz J. The quest for better understanding of HLA-disease association: scenes from a road less travelled by. Discov Med. 2013; 16(87): 93-101.

13. Lucas M, Karrer U, Lucas A, et al. Viral escape mechanisms--escapology taught by viruses. Int J Exp Pathol. 2001; 82 (5): 269-86.

14. Alcami A, Koszinowski UH. Viral mechanisms of immune evasion. Trends Microbiol. 2000; 8(9): 410-8.

15. Georgopoulos AP, James LM, Mahan MY, et al. Reduced Human Leukocyte Antigen (HLA) protection in Gulf War Illness (GWI) EBioMedicine. 2016; 3: 79-85.

16. Bettencourt A, Carvalho C, Leal B, et al. The protective role of HLADRB1*13 in autoimmune dis eases. J Immunol Res. 2015; 948723. http://dx.doi.org/10.1155/2015/948723.

17. Pereyra F, Jia X, et al. International HIV Controllers Study, The major genetic determinants of HIV-1 control affect HLA class I peptide presentation. Science. 2010; 330: 1551-7.

18. Goulder PJ, Walker BD. HIV and HLA class I: an evolving relationship. Immunity. 2012; 37: 426-40.

19. Singh R, Kaul R, Kaul A, et al. A comparative review of HLA associations with hepatitis $B$ and $C$ viral infections across global populations. World J Gastroenterol. 2007; 13(12): 1770-87.

20. Hill AV, Allsopp CE, Kwiatkowski D, et al. Common West African HLA antigens are associated with protection from severe malaria. Nature. 1991; 352: 595-600.

21. James LM, Christova P, Engdahl BE, et al. Human leukocyte antigen (HLA) and Gulf War Illness (GWI): HLA-DRB1*13:02 spares subcortical atrophy in Gulf War veterans. EBioMedicine. 2017; 26: 126-131.
22. James LM, Christova P, Lewis SM, et al. Protective Effect of Human Leukocyte Antigen (HLA) Allele DRB1* 13: 02 on Age-Related Brain Gray Matter Volume Reduction in Healthy Women. EBioMedicine. 2018; 29: 31-7.

23. James LM, Dolan S, Leuthold AC, et al. The effects of human leukocyte antigen DRB1* 13 and apolipoprotein $\mathrm{E}$ on age-related variability of synchronous neural interactions in healthy women. EBioMedicine. 2018; 35: 288-94.

24. Furukawa H, Oka S, Tsuchiya N, et al. The role of common protective alleles HLA-DRB1*13 among systemic autoimmune diseases. Genes Immun. 2017; 18: 1-7.

25. van der Woude D, Lie BA, Lundström E, et al. Protection against anti-citrullinated protein antibody-positive rheumatoid arthritis is predominantly associated with HLA-DRB1*1301: a meta-analysis of HLA-DRB1 associations with anti-citrullinated protein antibodypositive and anti-citrullinated protein antibody-negative rheumatoid arthritis in four European populations. Arthritis Rheum. 2010; 62: 1236-1245.

26. Fainboim L, Canero VMC, Marcos CY, et al. Protracted, but not acute, hepatitis a virus infection is strongly associated with HLA-DRB*1301, a marker for pediatric autoimmune hepatitis. Hepatology. 2001; 33: 1512-1517.

27. Institute of Medicine National Research Council. Gulf War and Health Volume 1. Depleted Uranium, Pyridostigmine Bromide, Sarin, and Vaccines. Washington, DC: National Academies Press, 2000.

28. Georgopoulos AP, James LM, Carpenter AF, et al. Gulf War illness (GWI) as a neuroimmune disease. Exp Brain Res. 2017; 235(10): 3217-3225.

29. White RF, Steele L, O'Callaghan JP, et al. Recent research on Gulf War illness and other health problems in veterans of the 1991 Gulf War: effects of toxicant exposures during deployment. Cortex. 2016; 74: 449-475.

30. Fukuda K, Nisenbaum R, Stewart G, et al. Chronic multisymptom illness affecting Air Force veterans of the Gulf War. JAMA. 1998; 280: 981-988.

31. Steele L. Prevalence and patterns of Gulf War illness in Kansas veterans: association of symptoms with characteristics of person, place, and time of military service Am J Epidemiol. 2000; 152: 9921002.

32. Broderick G, Ben-Hamo R, Vashishtha S, et al. Altered immune pathway activity under exercise challenge in Gulf War Illness: an exploratory analysis. Brain Behav Immun. 2013; 28: 159-169.

33. Hotopf M, David A, Hull L, et al. Role of vaccinations as risk factors for ill health in veterans of the Gulf war: cross-sectional study. BMJ. 2000; 320: 1363-1367.

34. Johnson GJ, Slater BC, Leis LA, et al. Blood biomarkers of chronic inflammation in Gulf War Illness. PLoS One. 2016; 11(6): e0157855

35. Esposito P, Gheorghe D, Kandere K, et al. Acute stress increases permeability of the blood-brain-barrier through activation of brain mast cells. Brain Res. 2001; 888: 117-127.

36. Christova P, James LM, Engdahl BE, et al. Subcortical brain atrophy in Gulf War Illness. Exp Brain Res. 2017; 235: 2777-2786.

37. Raz N, Rodrigue KM. Differential aging of the brain: patterns, cognitive correlates and modifiers Neurosci Biobehav Rev. 2006; 30: 730-748.

38. Enzinger C, Fazekas F, Matthews PM, et al. Risk factors for progression of brain atrophy in aging: six-year follow-up of normal subjects. Neurology. 2005; 64(10): 1704-11.

39. Baker DJ, Petersen RC. Cellular senescence in brain aging and neurodegenerative diseases: evidence and perspectives. J Clin Invest. 2018; 128(4): 1208-1216. 
40. Kirkland JL, Tchkonia T. Cellular senescence: a translational perspective. EBioMedicine. 2017; 21: 21-8.

41. Poulain M, Herm A, Pes G. The Blue Zones: areas of exceptional longevity around the world. Vienna Yearbook of Population Research. 2013 Jan 1; 87-108.
42. Georgopoulos AP, Tsilibary EP, Souto EP, et al. Adverse effects of Gulf War Illness (GWI) serum on neural cultures and their prevention by healthy serum. J Neurol Neuromed. 2018; 3(2): 19-27.

43. Tsilibary CEP, Souto EP, James LM, et al. Human immunoglobulin G (IgG) neutralizes adverse effects of Gulf War Illness (GWI) serum in Neural cultures: Paving the way to immunotherapy for GWI. J Neurol Neuromed. 2018; 3(5): 23-28. 